Int. J. Morphol.,

34(1):365-370, 2016

\title{
El Dimorfismo Sexual en Distintas Relaciones Cráneo-Mandibulares
}

\author{
Sexual Dimorphism in Different Craneomandibular Relationships
}

Ana Bucchi*; Cristina Bucchi** \& Ramón Fuentes**

BUCCHI, A; BUCCHI, C. \& FUENTES, R. El dimorfismo sexual en distintas relaciones cráneo-mandibulares. Int. J. Morphol., 34(1):365-370, 2016.

RESUMEN: La estimación del sexo en base a restos esqueletales es uno de los principales objetivos de las ciencias forenses. Esta estimación se basa en las diferencias de forma y tamaño que existen entre mujeres y hombres (dimorfismo sexual). En este trabajo se analiza la asociación entre el dimorfismo sexual de la mandíbula y las distintas relaciones cráneo-mandibulares (ortognata, prognata y retrognata). Se analizaron 4 medidas faciales (altura facial, altura de la rama de la mandíbula, ancho mínimo de la rama mandibular, largo del cuerpo de la mandíbula) en tele-radiografías de 114 pacientes chilenos de sexo y relación cráneo-mandibular conocidos para evaluar si, al igual que en poblaciones de otras partes del mundo, estas son sexualmente dimórficas. Los resultados indican que con la excepción del largo del cuerpo de la mandíbula, las demás variables permiten una elevada clasificación correcta del sexo de los individuos (88,6\% de los casos). Se concluye que una relación cráneo-mandibular retrognata o prognata, no afecta la estimación confiable del sexo de los individuos de población chilena.

PALABRAS CLAVE: Prognatismo; Retrognatismo; Dimorfismo sexual; Cefalometría.

\section{INTRODUCCIÓN}

La estimación del sexo en base a restos esqueletales es uno de los objetivos principales de las ciencias forenses. El sexo forma parte del perfil biológico básico de un individuo, junto con la estatura, la ancestría o afinidad poblacional y la edad (Buikstra \& Ubelaker, 1994; Krenzer, 2006).

La estimación del sexo se basa en el dimorfismo sexual que existe entre hombres y mujeres, y se refiere a las diferencias morfológicas de los caracteres sexuales secundarios, definidos en la pubertad. Ejemplos de dimorfismo sexual son la estatura promedio y robusticidad ósea mayor en hombres en comparación a las mujeres.

El cráneo, después de la pelvis, es la segunda estructura ósea más confiable para estimar el sexo de un individuo adulto, ya que logra estimar acertadamente entre el $80 \%$ y $92 \%$ de los casos (Krenzer; Iscan, 2005; Steyn \& Iscan, 1998). Sin embargo, es necesario conocer previamente los parámetros morfológicos específicos de la población a la que pertenecen los individuos, debido a que el dimorfismo sexual no es idéntico en todos los grupos humanos ni permanece constante en el tiempo (i. e. Garvin et al., 2014;
Kranioti \& Apostol, 2015; Wärmländer \& Sholts, 2011). La fiabilidad de estas estimaciones es mayor cuando existe un conocimiento sobre la morfología y el tamaño de los huesos de la población de interés y sobre las variables que están relacionadas con su variabilidad.

Diversos estudios han señalado que la altura facial, largo del cuerpo de la mandíbula y altura y ancho de la rama de la mandíbula son variables sexualmente dimórficas (Suazo et al., 2009; Binnal \& Devi, 2012; Baccetti et al., 2005), es decir, que la morfología mandibular varía según el sexo (i.e. Al-shamout et al., 2012; Suazo \& Zavando, 2012; Kharoshah et al., 2010; Loth \& Henneberg, 1996; Franklin et al., 2007), por lo que es utilizada como parámetro para la estimación del sexo en ciencias forenses (Krenzer; Buikstra \& Ubelaker).

Sin embargo, existen variables que influyen en el dimorfismo sexual mandibular, como el tamaño corporal (Kimmerle et al., 2008), la simetría de la cara (Little et al., 2008), y la afinidad poblacional (Franklin et al.). Así mismo, existen un número desconocido de variables relacionadas con el dimorfismo sexual de la mandíbula, algunas de

\footnotetext{
* Máster en Arqueología del Cuaternario y Evolución Humana, Universitat Rovira i Virgili, Tarragona, España

${ }^{*}$ Centro de Investigación en Ciencias Odontológicas (CICO), Facultad de Odontología, Universidad de La Frontera, Temuco, Chile.
} 
las cuales podría ser necesario considerar antes de hacer una estimación del sexo de un individuo. Una de estas es la relación que pueda tener con la mandíbula con respecto al cráneo (ortognata, prognata y retrognata), ya que se ha establecido previamente que existen diferencias significativas en determinadas mediciones mandibulares de individuos con clase III esqueletal (prognatismo) y el género al que pertenecen (Kuramae et al., 2005). Los hombres caucásicos prognáticos presentan mayores longitudes en la mandíbula y el maxilar, así como alturas faciales mayores que las mujeres (Baccetti et al.). Es necesario investigar la relación existente entre el dimorfismo sexual y las demás relaciones cráneo-mandibulares en población chilena.

El objetivo de este artículo fue establecer si una relación cráneo-mandibular retrognata o prognata altera la correcta identificación del sexo en un grupo de individuos chilenos.

\section{MATERIAL Y MÉTODO}

Este es un estudio descriptivo transversal. Se analizaron todas las telerradiografías de perfil digitales pertenecientes a un centro radiográfico universitario de la ciudad de Temuco, Chile, tomadas durante Junio y Agosto de 2014, previa firma del consentimiento informado por parte del paciente. Las radiografías fueron tomadas con la técnica estándar con un ortopantomógrafo (Pax Zenith, Vatech, Korea, 2011).

Se aplicaron los siguientes criterios de inclusión de la muestra:

-Telerradiografías de pacientes con género conocido.

- Telerradiografías de pacientes de 18 o más años.

-Telerradiografías de pacientes con soporte dentario posterior bilateral y presencia de los 4 incisivos mandibulares.

-Telerradiografías de pacientes con dientes en oclusión al momento de la toma radiográfica.

-Telerradiografías de pacientes sin evidencia de cirugía ortognática (placas de titanio), fractura o patología del hueso mandibular, nasal o frontal.

-Telerradiografías en que los puntos cefalométricos $\mathrm{S}, \mathrm{N}$ y B fueran claramente distinguibles.

Análisis cefalométrico. Las telerradiografías fueron analizadas mediante el software ImageJ (Versión 1.48, Schneider, et al., 2012). Posterior a la estandarización del tamaño de la radiografía, se midió el ángulo SNB, siendo este el ángulo formado por la línea que unen el punto $\mathrm{S}$ (silla turca) con el punto $\mathrm{N}$ (nasion) y la línea que une el punto $\mathrm{N}$ (nasion) y el punto B (base alveolar mandibular) (Fig. 1), según métodos previamente descritos (Sandoval et al., 2011). Un ángulo $80 \pm 2^{\circ}$ fue considerado como mandíbula ortognática, un ángulo superior a $82^{\circ}$ como mandíbula retrognática y un ángulo inferior a $78^{\circ}$ como mandíbula retrognática (Gregoret, 1997).

Las mediciones fueron realizadas por un único operador en dos oportunidades. Se calculó el coeficiente Kappa de Cohen, con el $10 \%$ de la muestra (11 telerradiografías).

Medición de variables dimórficas en las telerradiografías. Todas las medidas se llevaron a cabo por el mismo investigador.

En cada telerradiografía se midió (Fig. 2):

- La altura facial, medida desde Nasion (N) hasta Gnation (Gn).

- La altura de la rama de la mandíbula medida desde Gonion (Go) hasta Condilion (Co).

- El ancho mínimo de la rama mandibular.

- El largo del cuerpo de la mandíbula desde Gonion (Go) hasta Gnation (Gn).

Análisis estadístico. Se realizó el análisis de MANOVA para establecer diferencias significativas entre el sexo y todas las variables (altura facial, largo del cuerpo de la mandíbula y altura y ancho de la rama de la mandíbula), para cada relación cráneo-mandibular por separado. Se realizó otro MANOVA para evaluar si existen diferencias significativas entre cada relación cráneo-mandibular (ortognata, retrognata, prognata) y las variables estudiadas (altura facial, largo del cuerpo de la mandíbula y altura y ancho de la rama de la mandíbula), sin clasificarlas por sexo.

Posteriormente, se realizó un análisis de componentes principales, una técnica multivariada que permite explorar las relaciones entre todas las variables estudiadas. El análisis de componentes principales crea nuevas variables en base al conjunto de variables originales, con el objetivo de resumir los principales patrones de variación de los datos. Estas nuevas variables se llaman en orden de importancia "primer componente principal", que explica la mayor varianza de los datos, seguido de los demás componentes principales.

Por último, se realizó una regresión logística para evaluar cuán adecuadas son las variables en estudio para distinguir entre sexos. Se utilizaron los software estadísticos SPSS (versión 20.0, Chicago, IL) y Past (versión 2.17c, Hammer et al., 2013). Un umbral de 0,05 fue escogido como significancia estadística. 


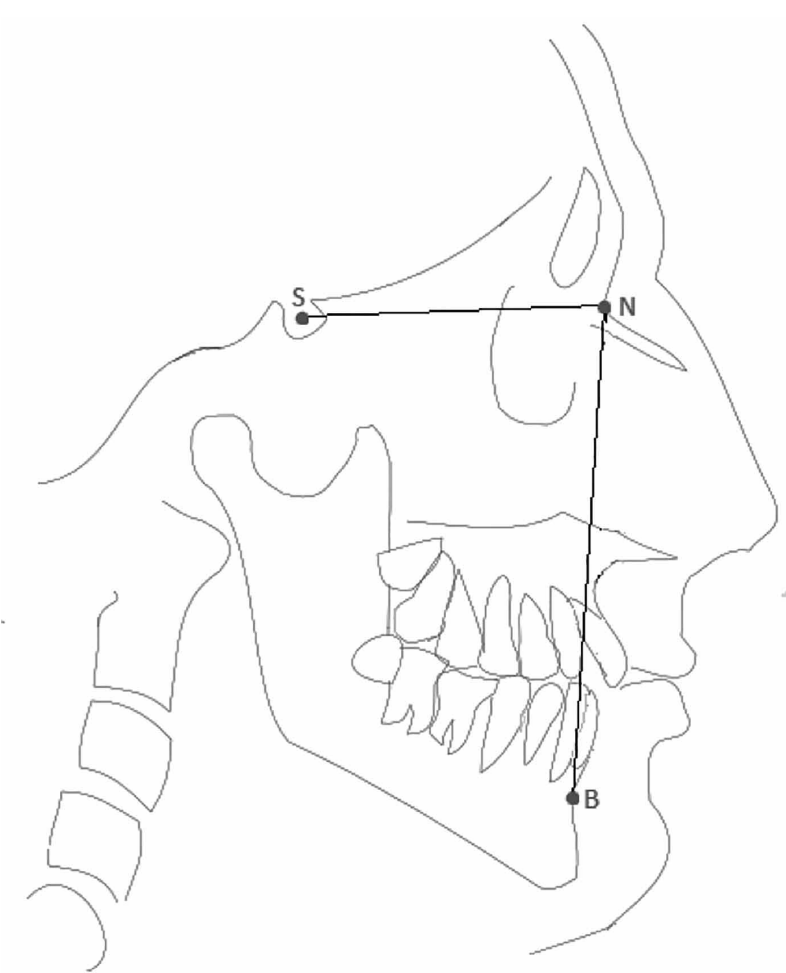

Fig. 1. Ángulo SNB para determinar la relación cráneo-mandibular (prognata, retrognata, ortognata). Un ángulo $80 \pm 2^{\circ}$ fue considerado como mandíbula ortognática, un ángulo superior a $82^{\circ}$ como mandíbula retrognática y un ángulo inferior a $78^{\circ}$ como mandíbula retrognática.

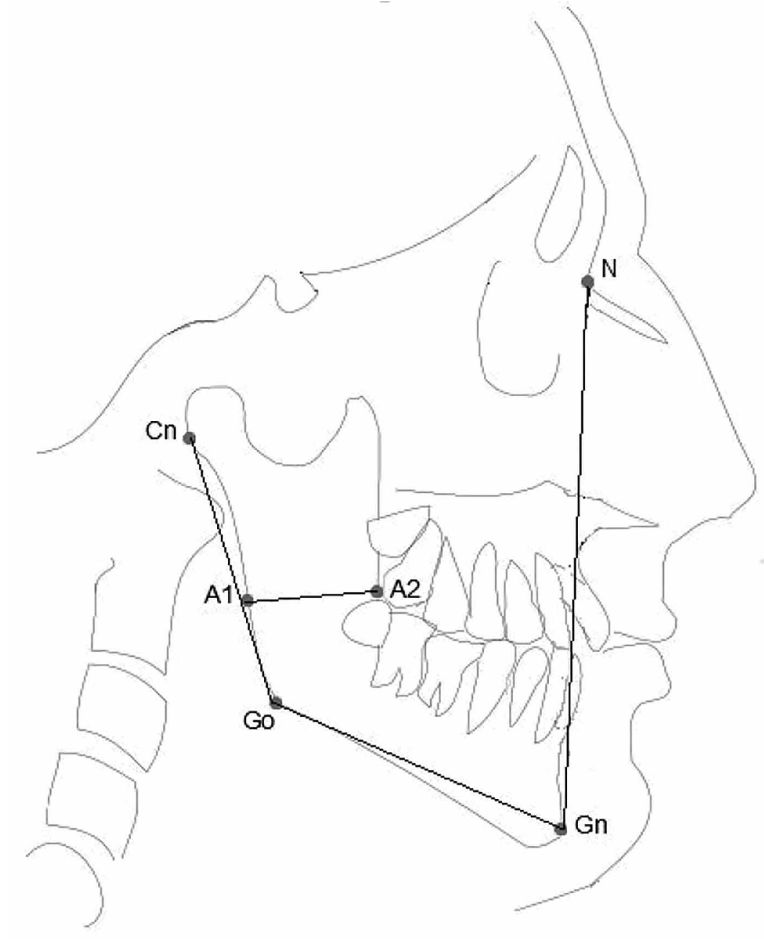

Fig. 2. Variables dimórficas medidas: Altura facial (N-Gn), Altura de la rama de la mandíbula (Cn-Go), Ancho mínimo de la rama mandibular (A1-A2) y largo de la mandíbula (Go-Gn)

\section{RESULTADOS}

La muestra final estuvo conformada por 114 telerradiografías, de las cuales 38 pertenecen a pacientes ortognáticos, 36 pacientes retrognáticos y 40 pacientes prognáticos. El coeficiente Kappa de Cohen para la clasificación de individuos en ortogantos, retrognatos o prognatos mostró que hubo concordancia entre la medición del tiempo 0 y tiempo $1(\mathrm{p}=0,001)$ y la fuerza de esta concordancia fue clasificada como "muy buena" $(0,81)$. En la Tabla I se describen los datos demográficos de las cefalometrías analizadas y su distribución por sexo.

Las variables dimórficas estudiadas muestran diferencias significativas según las relaciones cráneo- mandibulares, es decir, los pacientes ortognáticos, retrognáticos y prognáticos muestran diferencias significativas según las 4 variables en estudio.

Se encontraron diferencias estadísticamente significativas cuando se analizaron las variables dimórficas para hombres y mujeres para cada relación cráneo-mandibular por separado.

El gráfico de componentes principales (Fig. 3) muestra que el primer componente principal, que explica el $64 \%$ de la varianza total, separa a individuos femeninos (agrupadas a la derecha del gráfico) de los masculinos

Tabla I. Detalle de la información demográfica (sexo y edad) de los individuos según relación cráneo-mandibular.

\begin{tabular}{|c|c|c|c|c|c|c|}
\hline \multirow{2}{*}{$\begin{array}{l}\text { Relación cráneo- } \\
\text { mandibular }\end{array}$} & \multirow{2}{*}{ n } & \multicolumn{2}{|c|}{ Sexo } & \multirow{2}{*}{$\begin{array}{l}\text { Promedio } \\
\text { edad (DE) }\end{array}$} & \multicolumn{2}{|c|}{ Promedio edad (DE) según sexo } \\
\hline & & Hombres (n) & Mujeres (n) & & Hombres & Mujeres \\
\hline Ortognáticos & 38 & 16 & 22 & $26,8( \pm 9,8)$ & $25,6( \pm 8,1)$ & $27,7( \pm 11)$ \\
\hline Retrognáticos & 36 & 14 & 22 & $25,3( \pm 7,6)$ & $23,2( \pm 4,6)$ & $26,6( \pm 8,8)$ \\
\hline Prognáticos & 40 & 20 & 20 & $26,02( \pm 8,1)$ & $26,05( \pm 7,9)$ & $26( \pm 8,4)$ \\
\hline Total & 114 & 43 & 61 & $25,9( \pm 8,9)$ & $24,4( \pm 7,1)$ & $26,9( \pm 9,9)$ \\
\hline
\end{tabular}




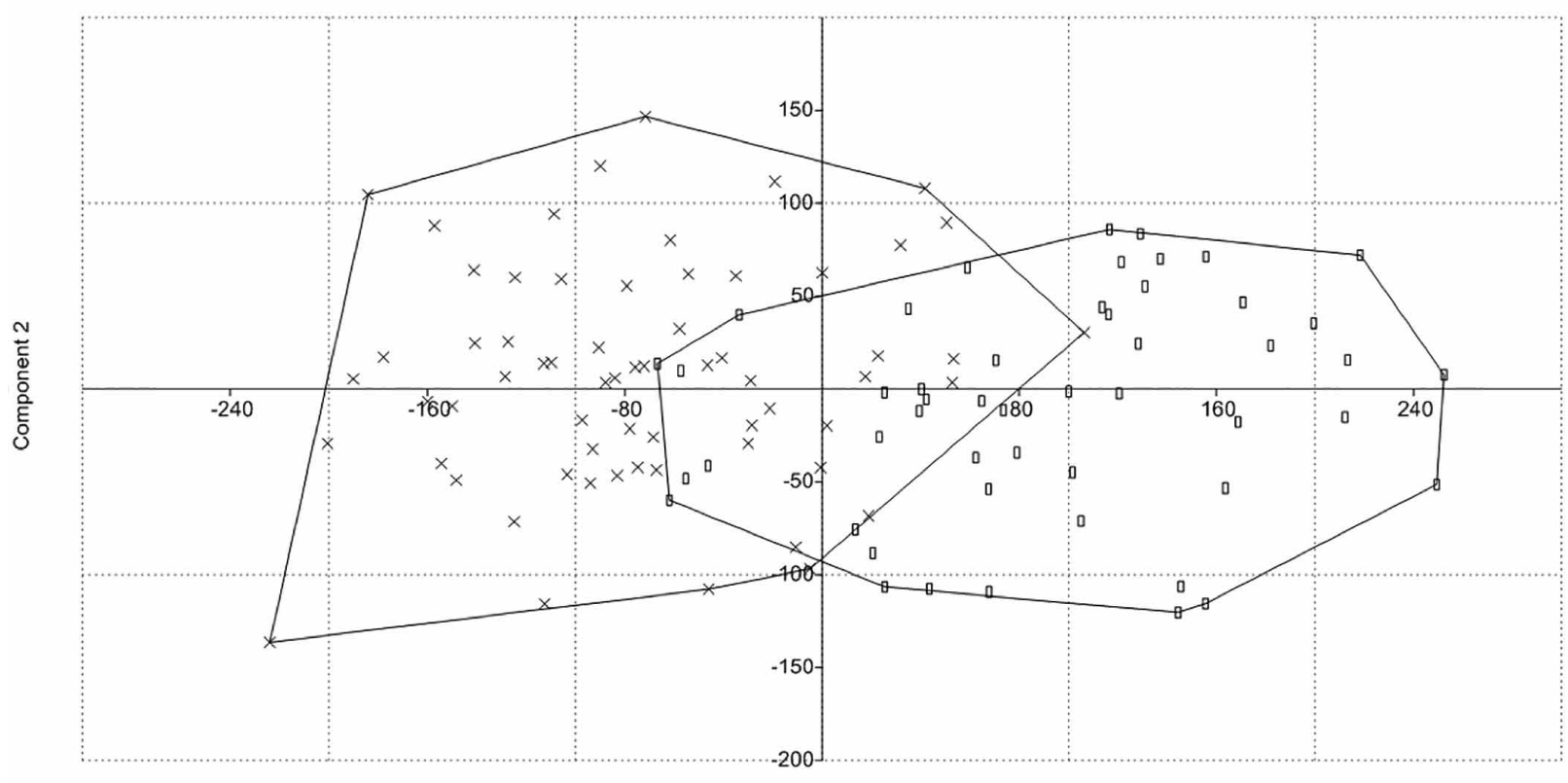

Component 1

Fig. 3. Análisis de componentes principales de todos los individuos de la muestra. El primer componente (eje X) explica el $64 \%$ de la varianza y el segundo (eje Y), el 18,4\%. Los círculos encierran a los individuos de cada sexo (femenino con una equis y masculino con rectángulos).

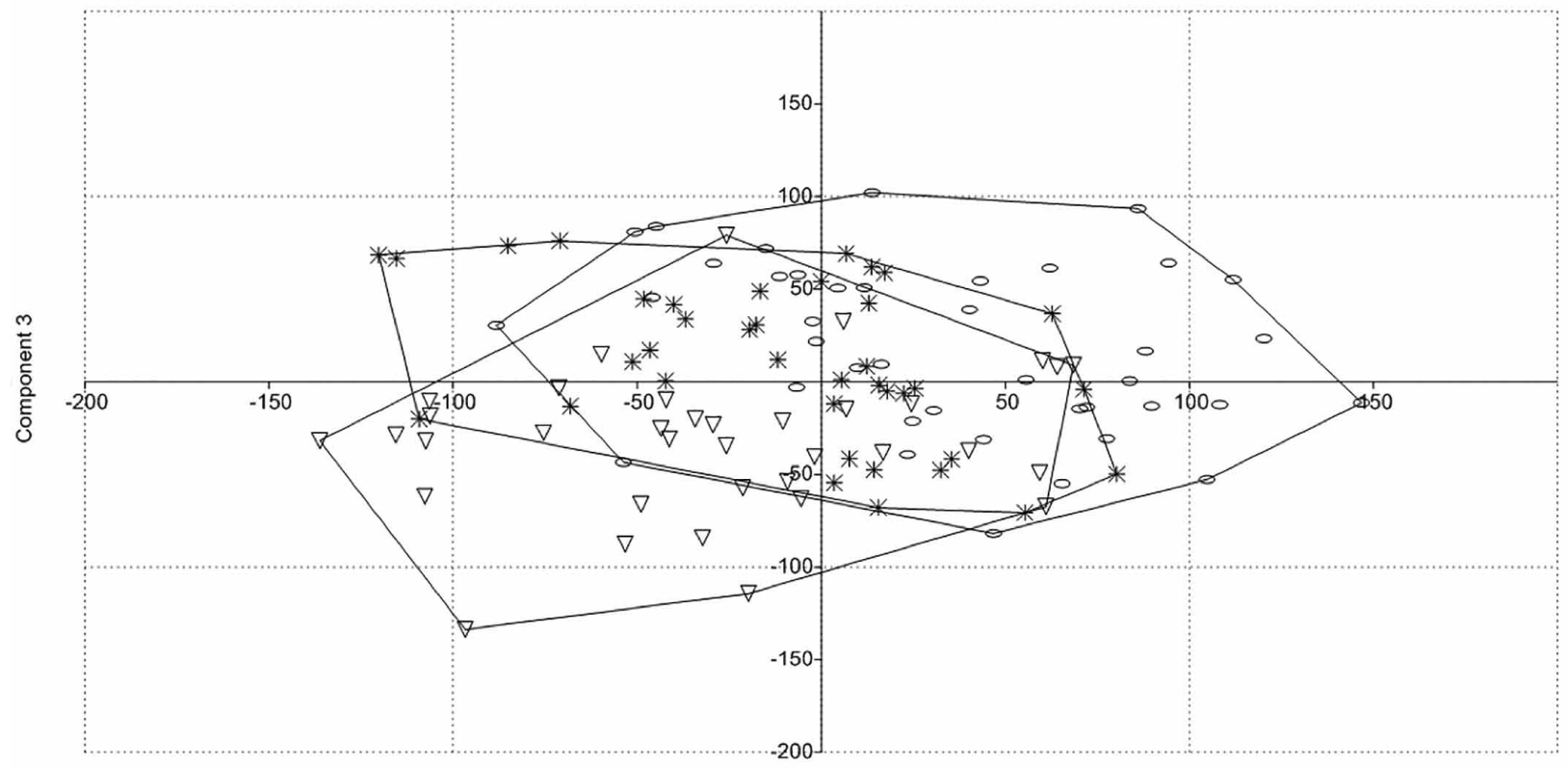

Component 2

Fig. 4. Análisis de componentes principales de todos los individuos de la muestra. El segundo componente (eje X) explica el 18,4 $\%$ de la varianza y el tercero (eje Y), el 11,9\%. Los círculos encierran a los individuos según su relación cráneo-mandibular (ortognáticos representados con asteriscos, prognáticos con óvalos y retrognáticos con triángulos).

(agrupados a la izquierda del gráfico). Esta variable indica que lo que separa a hombres de mujeres es una diferencia de tamaño y forma; los primeros tienden a tener mayores alturas faciales. Sin embargo, un aumento en la altura facial no implica un aumento proporcional de las demás va- riables, lo que causa una diferencia de forma. El largo del cuerpo de la mandíbula, la altura de la rama y el ancho de la rama son también mayores en hombres que en mujeres, pero no aumentan de manera proporcional a la altura facial. 
El segundo componente, que explica el $18,4 \%$ de la varianza, indica que la segunda variación importante en los datos es la tendencia de los individuos con mayores longitudes del largo de la mandíbula a tener las longitudes de las demás variables desproporcionadamente pequeñas. A diferencia de lo que ocurre con el primer componente, en el segundo no hay una clara diferencia debida al sexo, y tampoco debida a la relación cráneo mandibular, aunque indica una diferencia sutil entre prognáticos y retrognáticos, separados principalmente por las longitudes del ancho de la rama, mayores en promedio en los primeros que en los segundos. Los ortognatos se ubican entre ambos grupos (Fig. 4).

El modelo de la regresión logística para todos los individuos de la muestra es significativo $(\mathrm{p}<0,05)$ y tuvo una bondad de ajuste de 0,76 . Bajo este modelo se clasificaron correctamente el $88,6 \%$ de los casos según sexo, es decir, a 101 de los 114 pacientes. De las 4 variables en estudio, solo una, el largo del cuerpo de la mandíbula, resultó no significativa $(p>0,05)$. Todas las demás tuvieron pesos muy similares en la ecuación (coeficientes logísticos). Se obtuvieron resultados similares al hacer la regresión para cada relación cráneo-mandibular por separado, con porcentajes correctamente clasificados del 97,4\% para ortognáticos, 91,7 $\%$ para retrognáticos y $87,5 \%$ para prognáticos. En ninguno de estos últimos tres modelos las variables horizontales (ancho de la rama y largo del cuerpo mandibular) resultaron significativos.

\section{DISCUSIÓN}

El objetivo de este trabajo fue evaluar la asociación entre el dimorfismo sexual de la mandíbula y las distintas relaciones cráneo-mandibulares y de esta manera concluir si son variables que necesitan ser consideradas al utilizar la mandíbula para estimar el sexo de un individuo. Las variables dimórficas estudiadas, como lo son la altura facial, largo del cuerpo de la mandíbula y altura y ancho mínimo de la rama de la mandíbula, varían significativamente entre las distintas relaciones cráneo-mandibulares y también entre los sexos.

Los resultados confirman que en individuos de sexo conocido, las variables identificadas por otros estudios como sexualmente dimórficas (Binnal \& Devi; Baccetti et al.), lo son también para población chilena.

Una diferencia importante en las mediciones mandibulares entre hombres y mujeres es el tamaño y la forma. Es relevante que las variables verticales (altura facial y altura de la rama) varían más que las variables horizontales (ancho de la rama y largo del cuerpo de la mandí- bula), y son las que más ayudan a distinguir hombres de mujeres. Por el contrario, las variables horizontales son las que explica en mayor medida las diferencias morfológicas entre las distintas relaciones cráneo-mandibulares. En conclusión, las variables que explican con mayor peso las diferencias morfológicas entre hombres y mujeres no son las mismas que explican las diferencias entre individuos con distintas relaciones cráneo-mandibulares. Esto puede explicar por qué en la muestra de 114 individuos ortognáticos, prognáticos y retrognáticos, el porcentaje de casos correctamente asignados a cada sexo en la regresión logística sea bastante alto $(88,6 \%)$, lo que sugiere que puede hacerse una estimación del sexo confiable independiente de la relación cráneo mandibular del individuo. La única variable analizada que se recomienda no considerar cuando se estima sexo y se desconoce la relación cráneo mandibular del individuo es el largo del cuerpo de la mandíbula.

Las relaciones entre el retrognatismo y prognatismo y las variables dimórficas de interés para las ciencias forenses no han sido habitualmente abordadas. Sin embargo, tanto el retrognatismo y prognatismo mandibular, como otras variaciones morfológicas, añaden variabilidad a los las medidas lineales, las que pueden estar afectando las estimaciones del perfil biológico de un individuo, por lo que requieren ser más estudiadas.

\section{AGRADECIMIENTOS}

A la Unidad de Radiología de la Facultad de Odontología de la Universidad de La Frontera, por la concesión de las radiografías.

BUCCHI, A; BUCCHI, C. \& FUENTES, R. Sexual dimorphism in different craneomandibular relationships. Int. J. Morphol., 34(1):365-370, 2016.

SUMMARY: Sex assessment using bone remains is one of the main goals of forensic sciences. This assessment is possible because of the morphological and size differences between women and men (sexual dimorphism). In this work we study the association between sexual dimorphism and the different positions of the mandible and skull (prognathism, retrognathism and orgotnatism). We analyze 4 facial measurements in 114 teleradiographies of Chilean patients with known sex and positional relationship of the mandible and cranium, to evaluate if, as in populations of other parts of the world, these are sexually dimorphic. The results indicate that, with the exception of mandible width, the rest of the variables allows a high correct classification of individuals by sex 
( $88.6 \%$ of the cases). We conclude that the relation between different positions of the mandible and skull does not affect a reliable sex assessment in Chilean population.

KEY WORDS: Prognathism; Retrognathism; Sexual dimorphism; Cephalometrics.

\section{REFERENCIAS BIBLIOGRÁFICAS}

Al-Shamout, R.; Ammoush, M.; Alrbata, R. \& Al-Habahbah, A. Age and gender differences in gonial angle, ramus height and bigonial width in dentate subjects. Pak. Oral Dental J., 32(1):81-7, 2012.

Baccetti, T.; Reyes, B. C., \& McNamara, J. A. Jr. Gender differences in Class III malocclusion. Angle Orthod., 75(4):510-20, 2005.

Binnal, A. \& Devi, B. K. Y. Identification of sex using lateral cephalogram: Role of cephalofacial parameters. J. Indian Acad. Oral Med. Radiol., 24(4):280-3, 2012.

Buikstra, J. E. \& Ubelaker, D. H. Standards for Data Collection from Human Skeletal Remains: Proceedings of a Seminar at the Field Museum of Natural History. Fayetteville, Arkansas Archeological Survey, 1994.

Franklin, D.; O’Higgins, P.; Oxnard, C. E. \& Dadour, I. Sexual dimorphism and population variation in the adult mandible : Forensic applications of geometric morphometrics. Forensic Sci. Med. Pathol., 3(1):15-22, 2007.

Garvin, H. M.; Sholts, S. B. \& Mosca, L. A. Sexual dimorphism in human cranial trait scores: effects of population, age, and body size. Am. J. Phys. Anthrop., 154(2):259-69, 2014.

Gregoret, J. Ortodoncia y Cirugía Ortognática. Diagnóstico y Planificación. Madrid, ESPAXS, 1997.

Hammer, Ø.; Harper, D. A. T. \& Ryan, P. D. PAST: Paleontological statistics software package for education and data analysis. Palaeontol. Electron., 4(1):9pp., 2013.

Iscan, M. Y. Forensic anthropology of sex and body size. Forensic Sci. Int., 147(2-3):107-12, 2005.

Kimmerle, E. H.; Ross, A. \& Slice, D. Sexual dimorphism in America: geometric morphometric analysis of the craniofacial region. J. Forensic Sci., 53(1):54-7, 2008.

Kharoshah, M. A.; Almadani O.; Ghaleb, S. S.; Zaki, M. K. \& Fattah, Y. A. Sexual dimorphism of the mandible in a modern Egyptian population. J. Forensic Leg. Med., 17(4):213-5, 2010.

Kranioti, E. F. \& Apostol, M. A. Sexual dimorphism of the tibia in contemporary Greeks, Italians, and Spanish: forensic implications. Int. J. Legal Med., 129(2):357-63, 2015.
Krenzer, U. Compendio de Métodos Antropológico Forenses para la Reconstrucción del Perfil Osteo-Biológico. Guatemala, Centro de Análisis Forense y Ciencias Aplicadas, 2006.

Kuramae, M.; Magnani, M. B. B. A.; Pereira Neto, J. S.; Nouer, D. F. \& Nouer P. R. A. Cephalometric evaluation of Class III malocclusion by a modified Tweed-Merrifield analysis. Braz. J. Oral Sci., 4(12):680-4, 2005.

Little, A. C.; Jones, B. C.; Waitt, C.; Tiddeman, B. P.; Feinberg, D. R.; Perrett, D. I.; Apicella, C. L. \& Marlowe, F. W. Symmetry is related to sexual dimorphism in faces: data across culture and species. PLoS One, 3(5):e2106, 2008.

Loth, S. R. \& Henneberg, M. Mandibular ramus flexure: a new morphologic indicator of sexual dimorphism in the human skeleton. Am. J. Phys. Anthropol., 99(3):473-85, 1996.

Sandoval, P.; García, N.; Sanhueza, A.; Romero, A. \& Reveco, R. Cephalometric measurements in lateral radiographs of fiveyear-old pre-schoolers in the city of Temuco. Int. J. Morphol., 29(4):1235-40, 2011.

Schneider, C. A.; Rasband, W. S. \& Eliceiri, K. W. NIH Image to ImageJ: 25 years of image analysis. Nat. Methods, 9:671-5, 2012.

Steyn, M. \& Iscan, M. Y. Sexual dimorphism in the crania and mandibles of South African whites. Forensic Sci. Int., 98(1):916, 1998.

Suazo Galdames, I. \& Zavando, D. Age effect in the morphological traits performance for sex determination in human skulls and mandibles. Int. J. Morphol., 30(1):296-301, 2012.

Suazo Galdames, I. C.; Zavando Matamala, D. A. \& Smith, R. L. Sex determination in mandibles in the first year of life by a quantitative approach. Int. J. Morphol., 27(1):113-6, 2009.

Wärmländer, S. K. \& Sholts, S. B. Sampling and statistical considerations for the Suchey-Brooks method for pubic bone age estimation: implications for regional comparisons. Sci. Justice, 51(3):131-4, 2011.

Dirección para Correspondencia:

Ana María Bucchi Morales

Comte de Rius 10, piso 2D

Código Postal: 43003

Tarragona

ESPAÑA

Email: anabucchi@gmail.com

Recibido : 03-09-2015

Aceptado: 26-10-2015 